\title{
Co-rich cobalt platinum nanowire arrays: Effects of annealing
}

\author{
Wuxia Li, Yong Peng, Grenville A. Jones, and Tiehan H. Shen ${ }^{\text {a) }}$ \\ Joule Physics Laboratory, Institute for Materials Research, University of Salford, Salford, \\ M5 4WT, United Kingdom \\ Geoff Hill \\ EPSRC National Centre for III-V Technologies, University of Sheffield, Mappin Street, \\ Sheffield, S1 3JD, United Kingdom
}

(Received 17 May 2004; accepted 10 November 2004; published online 14 January 2005)

\begin{abstract}
The effects of annealing on the crystal structure and magnetic properties of Co-rich cobalt platinum nanowire arrays embedded in anodic aluminium oxide membranes have been investigated. For this purpose, a rapid thermal annealing to temperatures of $300{ }^{\circ} \mathrm{C}$ to $800{ }^{\circ} \mathrm{C}$ has been used. Transmission electron microscopy and scanning electron microscopy show that the nanowires have a mean diameter of $14 \mathrm{~nm}$ and an estimated wire density of $7.8 \times 10^{10} \mathrm{~cm}^{-2}$. From x-ray diffraction patterns, we find that the nanowires are hcp and possess a preferred texture in which the $c$ axis of the grains tends to lie along the major axis of the wire. Vibrating sample magnetometry measurements indicate that the easy axis is along the nanowire axis direction. Hysteresis loops, saturation magnetization, squareness ratio $\left(M_{\mathrm{r}} / M_{\mathrm{S}}\right)$, and coercivity (perpendicular and parallel to the nanowire axis) have all been investigated as a function of the annealing temperature $\left(T_{\mathrm{A}}\right)$. Coercivity parallel to the wire axis first increases with $T_{\mathrm{A}}$, attains a maximum at $600{ }^{\circ} \mathrm{C}$ (which is $150 \%$ of the as-deposited sample), and then decreases. By contrast there is relatively little change in the coercivity measured perpendicular to the wires. The saturation magnetization for the as-deposited sample is $1360 \mathrm{emu} / \mathrm{cc}$ and remains almost constant for annealing temperatures up to $500{ }^{\circ} \mathrm{C}$ : for $T_{\mathrm{A}}>500{ }^{\circ} \mathrm{C}$ it decreases significantly. The maximum $\left(M_{\mathrm{r}} / M_{\mathrm{s}}\right)$ ratio attained in this study is 0.99 , the highest value reported thus far for cobalt platinum alloy nanowires. The data suggest that these materials are potential candidates for high-density magnetic recording media. (C) 2005 American Institute of Physics. [DOI: 10.1063/1.1846941]
\end{abstract}

\section{INTRODUCTION}

Recent progress in the preparation and characterization of the properties of CoPt and FePt thin films and nanoparticles has been largely motivated by their potential applications in high-density magnetic and magneto-optical recording. ${ }^{1-4}$ Requirements for higher magnetic recording density with low noise demand that materials consist of magnetically isolated grains less than $10 \mathrm{~nm}$ in diameter with high magnetocrystalline anisotropy; the latter inhibits thermal fluctuations that tend to destabilize the magnetization of the recorded bits. ${ }^{5}$ Intensive studies have been conducted in order to attain the combined goals of a high areal density of bits and adequate thermal stability. ${ }^{6-8}$ One-dimensional nanowire arrays based on anodic aluminum oxide (AAO) present an attractive potential medium because when deposited the wires comprise a highly ordered pattern of magnetically isolated units. ${ }^{9-11}$ Although only a few reports are current for CoPt nanowire materials, ${ }^{12-14}$ the highest squareness ratio of CoPt nanowire embedded in AAO with a wire diameter of $80 \mathrm{~nm}$ has been claimed by Yasui et al. as $0.96 .^{14}$

We have recently prepared electrodeposited $\mathrm{Co}_{(1-x)} \mathrm{Pt}_{x}$ nanowire arrays having a factor of 6 smaller wire diameter compared with wires reported by Yasui et al. ${ }^{14}$ As a first step in this study, we concentrate on $\mathrm{Co}_{96} \mathrm{Pt}_{4} \mathrm{Co}$-rich nanowire arrays that were annealed up to $800{ }^{\circ} \mathrm{C}$ under the protection

${ }^{a)}$ Electronic mail: t.shen@salford.ac.uk of nitrogen in a rapid thermal annealing system. The magnetic properties and nanostructure of these Co-rich nanowire arrays have been investigated as a function of annealing temperature.

\section{EXPERIMENT}

Specimens for this study were fabricated by electrodepositing Co-Pt alloy on to anodized AAO templates; the detailed procedure and the properties of the AAO templates used have been published elsewhere. ${ }^{15}$ The electrolytes in this work consisted of $0.5 \mathrm{~g} / 1 \mathrm{PtCl}_{4}, 96 \mathrm{~g} / 1 \mathrm{CoSO}_{4} \cdot 7 \mathrm{H}_{2} \mathrm{O}$ and $42 \mathrm{~g} / 1 \mathrm{H}_{3} \mathrm{BO}_{3}$. The electrolysis was carried out at room temperature for $40 \mathrm{~s}$ with an ac voltage (frequency $=200 \mathrm{~Hz}$ ) of $13 \mathrm{~V}$.

After electrodeposition the ordered nanowire arrays were separated from the Al substrates by immersion in a saturated $\mathrm{HgCl}_{2}$ solution. Nanowires embedded in AAO were annealed at different temperatures $\left(300{ }^{\circ} \mathrm{C}, 400{ }^{\circ} \mathrm{C}, 500{ }^{\circ} \mathrm{C}, 600{ }^{\circ} \mathrm{C}\right.$, $650{ }^{\circ} \mathrm{C}, 700{ }^{\circ} \mathrm{C}, 800{ }^{\circ} \mathrm{C}$ ) for $30 \mathrm{~s}$ under a nitrogen atmosphere. To measure the actual length and diameter of the nanowires, the AAO membranes were partially dissolved in aqueous $\mathrm{NaOH}$ solution. The free-standing wires were then cast on to carbon-coated copper TEM grids and dried in air before insertion into the TEM chamber. The chemical composition of the nanowire arrays was determined by energy dispersive $\mathrm{x}$-ray spectrometry (EDS) and the crystal structure of the as-deposited and annealed samples was investigated 


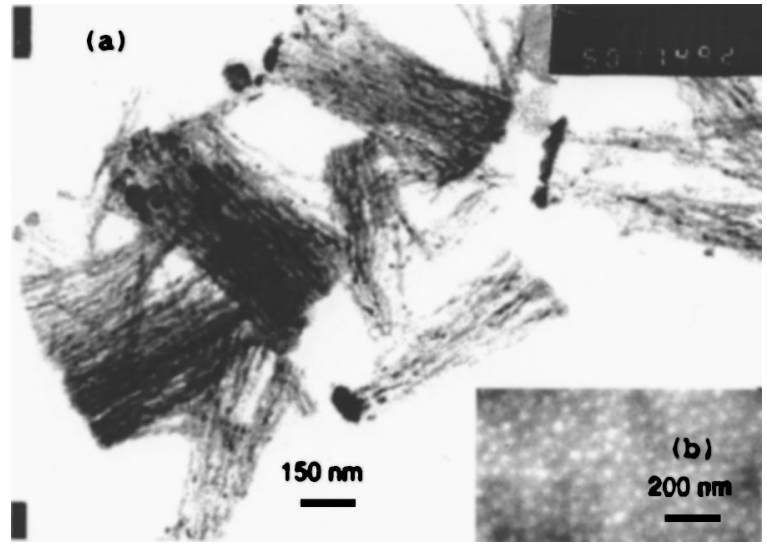

FIG. 1. (a) TEM image of $\mathrm{Co}_{96} \mathrm{Pt}_{4}$ nanowires freed from the $\mathrm{AAO}$ matrix; (b) SEM plan view of a mechanically polished sample of nanowires embedded in an AAO template prepared for chemical composition analysis.

by x-ray diffraction (XRD). The diffractometer was scanned at a rate of $0.1 \mathrm{deg} / \mathrm{min}$ in the range of $30 \mathrm{deg}<(2 \theta)$ $<90 \mathrm{deg}$. The optical absorbance [defined as $\log _{10}\left(I_{0} / I\right)$, where $I_{0}$ and $I$ are the intensities of the incident beam and transmitted beam, respectively] of some wire arrays was monitored with an HP 8452A spectrophotometer.

The magnetic properties of the samples were measured with a vibrating sample magnetometer (VSM). Hysteresis loops were obtained with the external field applied parallel, perpendicular, and at $45 \mathrm{deg}$ to the wire axis: these yielded values of coercivity $\left(H_{\mathrm{c}}\right)$, saturation magnetization $\left(M_{\mathrm{s}}\right)$, and squareness $\left(M_{\mathrm{r}} / M_{\mathrm{s}}\right)$.

\section{RESULTS}

Figure 1(a) is a TEM micrograph that demonstrates the typical topography of nanowires freed from an AAO template; the wires have an average diameter of $14 \mathrm{~nm}$ and length of $0.49 \mu \mathrm{m}$. Although the nanowire length and diameter are uniform, high magnification micrographs (not presented) reveal that an individual wire consists of elliptically shaped grains, a result similar to that reported by Peng et al. ${ }^{16}$

A "plan-view" SEM micrograph of a mechanically polished as-deposited $\mathrm{Co}_{96} \mathrm{Pt}_{4}$ nanowire array (prepared for EDS analysis) is presented in Fig. 1(b). This micrograph allowed the mean areal density of nanowires to be calculated as 7.8 $\times 10^{10} \mathrm{~cm}^{-2}$, a value suitable for high-density recording media. $^{17}$

The x-ray spectra obtained by the diffractometer are shown in Fig. 2(a). A clear distinction is observed between the as-deposited sample and the annealed samples insofar as the latter have more prominent peaks; this may be the result of stress relief or low-temperature grain growth. On the other hand, the spectra show no great changes with $T_{\mathrm{A}}$ in the range $300-600{ }^{\circ} \mathrm{C}$. This is perhaps surprising because, to the naked eye, the sample annealed at $600{ }^{\circ} \mathrm{C}$ had changed color slightly and seemed somewhat less opaque. The latter result is confirmed by absorbance spectra obtained over the ultraviolet and visible regions shown in Fig. 2(b). The decrease in
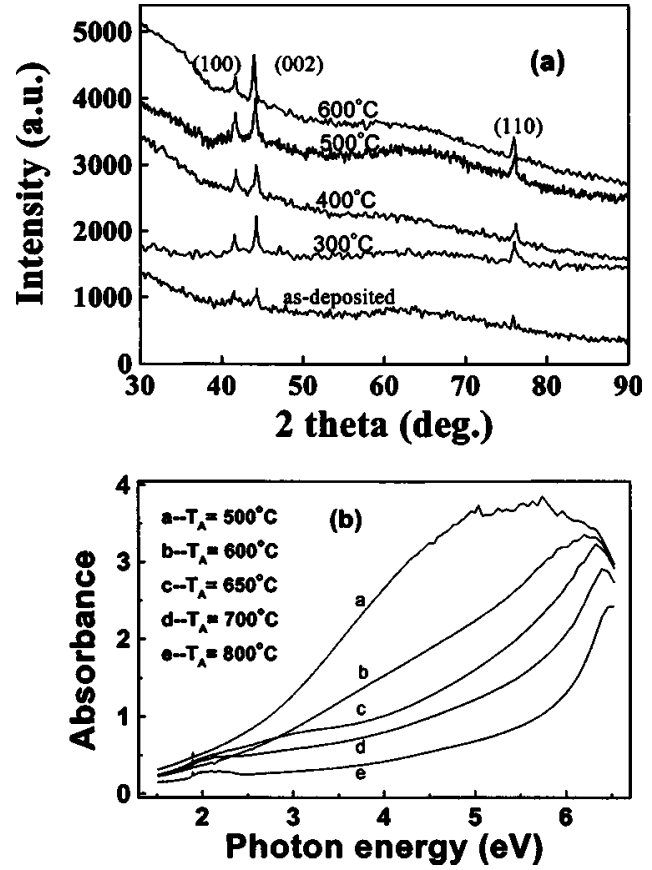

FIG. 2. (a) X-ray diffraction patterns of $\mathrm{Co}_{96} \mathrm{Pt}_{4}$ nanowire arrays as a function of $T_{\mathrm{A}}$ (for $T_{\mathrm{A}}$ up to $600{ }^{\circ} \mathrm{C}$ ); (b) visible and ultraviolet absorption spectra of samples annealed at $500{ }^{\circ} \mathrm{C}, 600{ }^{\circ} \mathrm{C}, 650{ }^{\circ} \mathrm{C}, 700{ }^{\circ} \mathrm{C}$, and $800{ }^{\circ} \mathrm{C}$.

absorbance (i.e., increased transmission of light) is clearly marked as the annealing temperature is increased. This is a feature to which we shall return below.

Returning to the x-ray spectra, analysis proved that the nanowire material comprises an hcp phase: the three identifiable peaks are (100), (002), and (110). The lattice parameters of the nanowires as calculated from Bragg's law are $a$ $=(2.516 \AA, 2.499 \AA)$ and $c=(4.080 \AA, 4.120 \AA)$ for the asdeposited sample and the sample annealed at $600{ }^{\circ} \mathrm{C}$, respectively. Comparison with the values for pure cobalt, namely $a=2.503 \AA$ and $c=4.060 \AA$, indicates that the lattice parameters are affected only minimally by the annealing procedure. The absence of a (101) peak, together with the relatively large magnitude of the (002) peak, indicate that the $c$ axis of the grains within a wire tends to lie parallel to the wire axis.

Fig. 3(a) shows the variation of $M_{\mathrm{s}}$ as a function of $T_{\mathrm{A}}$. The as-deposited sample has a value of $M_{\mathrm{s}}$ that is fairly close to that of bulk cobalt $(1420 \mathrm{emu} / \mathrm{cc})$. To begin with it remains constant with $T_{\mathrm{A}}$ but falls by about $10 \%$ following an annealing at $500{ }^{\circ} \mathrm{C}$. Thereafter, the saturation magnetization decreases more rapidly and samples annealed at $700{ }^{\circ} \mathrm{C}$ were effectively nonmagnetic at room temperature.

The VSM was also used to obtain coercivity values, and the results are shown in Fig. 3(b) for the external field applied parallel and perpendicular to the wire axis. There is very little variation of the latter with $T_{\mathrm{A}}$, a value of about 500 Oe being recorded. $H_{\mathrm{c}}$ values parallel to the wire axis are much larger (by a factor of at least 3 ) and, furthermore, some variation is discerned with $T_{\mathrm{A}}$. The as-deposited sample has an $H_{\mathrm{c}}$ of 1560 Oe: this increases until a maximum of $2500 \mathrm{Oe}$ is attained at $T_{\mathrm{A}}=600{ }^{\circ} \mathrm{C}$. Similar behavior was observed for the coercivity measured at $45 \mathrm{deg}$ to the wire axis. Hysteresis loops for an as-deposited and a sample 

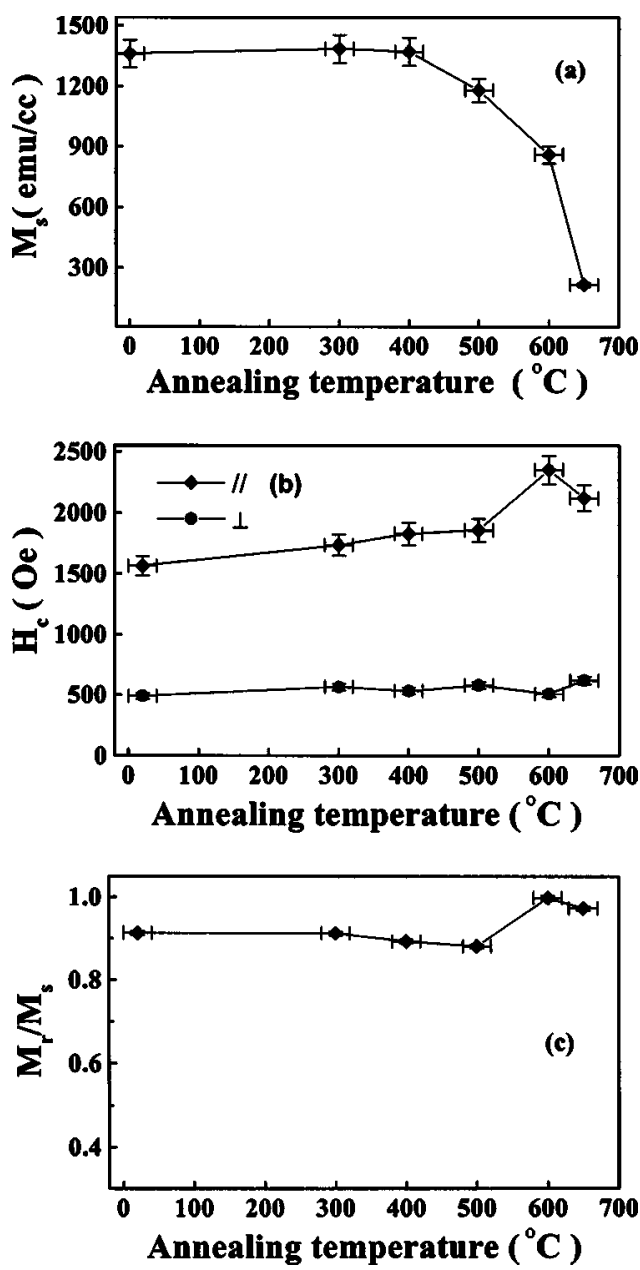

FIG. 3. Various magnetic properties measured as a function of annealing temperature $\left(T_{\mathrm{A}}\right)$. (a) saturation magnetization; (b) coercivity $H_{\mathrm{c}}$, with the external field parallel $(\bullet)$ and normal $(\bullet)$ to the wire axis; (c) $M_{\mathrm{r}} / M_{\mathrm{s}}$ with the field applied along the nanowire axis. All magnetic measurements performed at room temperature.

annealed at $600{ }^{\circ} \mathrm{C}$ are displayed in Fig. 4. Of note is the striking difference between the loops measured parallel and perpendicular to the wire axis: this will be discussed later. It is apparent that the annealing process does not radically alter the magnetic behavior apart from the increase in coercivity alluded to above. The squareness ratio reaches a maximum value of 0.99 for the loop traced along the major axis of wires annealed at $600{ }^{\circ} \mathrm{C}$.

\section{DISCUSSION AND CONCLUSIONS}

The x-ray data indicate that the nanowire arrays studied here comprise an hcp phase. The absence of a (101) peak means that the wires are textured. In fact, some uncertainty surrounds the texture of Co nanowires. Forster et al. ${ }^{18}$ in a study of $55 \mathrm{~nm}$ diameter wires, conclude that individual grains have the $c$ axis either parallel or normal to the wire axis; the volume fraction of the latter lay between $70 \%-90 \%$. By contrast, Henry et al. ${ }^{19}$ report that in small diameter $(<50 \mathrm{~nm})$ wires an alignment of the $c$ axis with the wire axis is preferred. Very recently, Darques et al..$^{20,21}$ have suggested that the orientation of the $c$ axis can be controlled by adjustment of the electroplating solution and the deposition condi-
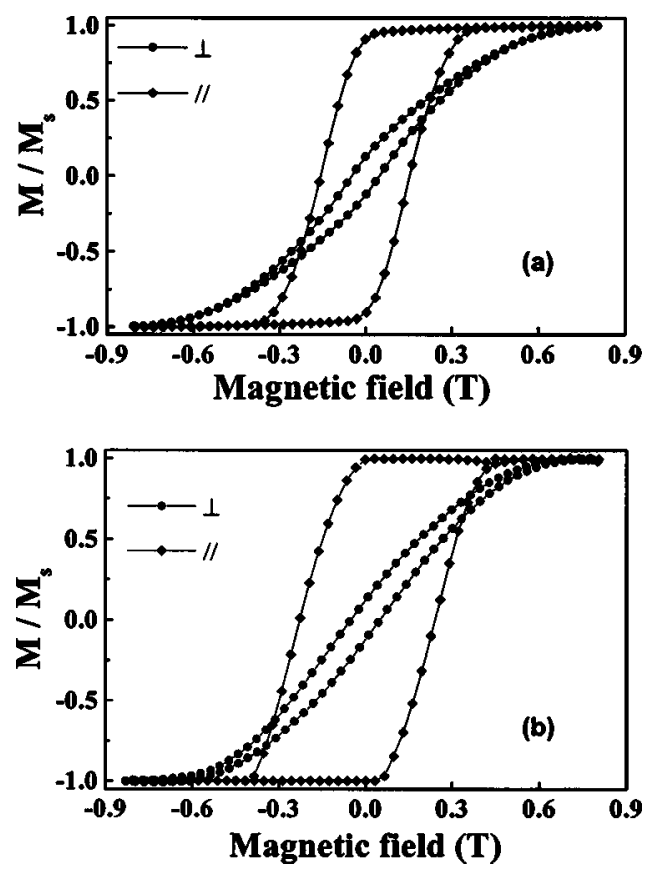

FIG. 4. $M / M_{s}-H$ loops of $\mathrm{Co}_{96} \mathrm{Pt}_{4}$ nanowire arrays measured with external field parallel ( ) and normal $(\bullet)$ to the wire axis: (a) as-deposited sample; (b) sample rapid thermally annealed at $600{ }^{\circ} \mathrm{C}$ for $30 \mathrm{~s}$.

tions. In our nanowire arrays the simultaneous presence of (100), (002), and (110) reflections precludes a single orientation of $c$ axis either parallel or normal to the wire axis. However, contrary to Forster et al. ${ }^{18}$ - and in light of the relative magnitude of the (002) peak-we believe that in these very fine wires $(14 \mathrm{~nm})$ there is a tendency for the $c$ axis to align with the wire axis. Such a result would concur with the finding of Henry et al. ${ }^{19}$ High-resolution TEM and diffraction might provide a definitive answer, but our instrument has failed to resolve the issue.

One of the most intriguing results of this study is the apparent discrepancy between the diminution in $M_{\mathrm{S}}$ for $T_{\mathrm{A}}$ $>400{ }^{\circ} \mathrm{C}$ but with no accompanying change in the $\mathrm{x}$-ray spectra (at least up to $600{ }^{\circ} \mathrm{C}$ ). Several mechanisms may be adduced for the fall in $M_{\mathrm{s}}$ value, but they must all involve a loss of free-magnetic cobalt atoms: for example, oxidation due to combination with oxygen ions either already present in the nanowires or as an impurity in the nitrogen atmosphere of the RTA chamber. Small amounts of oxide may not register on the diffractometer. Another possibility is that the wires melt during the rapid anneal and the nitrogen stream promotes the evaporation of Co atoms. It is known that the melting point of most metallic nanoparticles, nanorods, and nanowires is much less than that of bulk material. ${ }^{22,23}$ In an effort to confirm this hypothesis a small sample of nanowires was subject to differential scanning calorimetry (up to $600{ }^{\circ} \mathrm{C}$ ). No peak that could be attributed to melting was observed, although a small feature was detected at $420{ }^{\circ} \mathrm{C}$. We believe that this feature might be associated with the normal hcp/fcc phase change in cobalt.

Before discussing diffusion effects, it is pertinent to consider the possibility of an inhomogeneous distribution of Co atoms within the wires. Under certain circumstances, e.g., pulsed electrodeposition, the concentrations of the two ele- 
ments (Co and $\mathrm{Pt}$ ) can show a marked spatial preference: indeed, a "bamboo" structure can be obtained. ${ }^{24}$ However, it can be easily demonstrated for our samples that with an ac deposition frequency of $200 \mathrm{~Hz}$ and a deposition time of $40 \mathrm{~s}$ for a nanowire length of about $0.5 \mu \mathrm{m}$, then each cycle of growth represents an increment in wire length of about $0.06 \mathrm{~nm}$. Thus, any variations in concentration occurring within a growth cycle are at the sub-Ångstrom level. Effectively, the wires are compositionally homogeneous.

Even if a wire did possess concentration gradients on the nanometer scale, it is most improbable that intrawire redistribution of $\mathrm{Co}$ and $\mathrm{Pt}$ atoms alone could account for the dramatic fall in $M_{\mathrm{s}}$ in the range $400{ }^{\circ} \mathrm{C}<T_{\mathrm{A}}<650{ }^{\circ} \mathrm{C}$. At the Co-rich end of the Co-Pt solid solution, the saturation magnetization is proportional to Co content, and so realistically one would expect to find a maximum variation of order $4 \%$ in $M_{\mathrm{s}}$ for an alloy of macroscopic composition $\mathrm{Co}_{96} \mathrm{Pt}_{4}$. The most likely explanation for the decrease in net magnetic moment is that Co atoms diffuse from the nanowires (or react at the interface) into the alumina-lined pores and combine to form a nonmagnetic oxide of the type $(\mathrm{Al}, \mathrm{Co})_{2} \mathrm{O}_{3}$. Essentially, this is the process of calcination used commercially to create glazes in ceramics. Support for this proposition is drawn from color changes observed when samples are annealed. Thus, a sample remains brown when annealed up to $600{ }^{\circ} \mathrm{C}$, turns light brown at $650{ }^{\circ} \mathrm{C}$, then light gray at $700{ }^{\circ} \mathrm{C}$, and finally soft blue at $800{ }^{\circ} \mathrm{C}$. Physically this is accompanied by increased transparency to light [Fig. 2(b)]. It must be presumed that the number of $\mathrm{Co}$ atoms reacting at the lower temperature of $500{ }^{\circ} \mathrm{C}$, although sufficient to affect the saturation magnetization, fails to produce detectable changes in coloration. The x-ray spectra [Fig. 2(a)] reveal no peaks for $\mathrm{Al}_{2} \mathrm{O}_{3}$ : the oxide may be in a quasiamorphous state or perhaps present in too small an amount to register a signal. It is not surprising, therefore, that the reaction of $\mathrm{Co}$ with $\mathrm{Al}_{2} \mathrm{O}_{3}$ also does not produce significant changes in the spectra. In a detailed study of the $\mathrm{Co} / \mathrm{Al}_{2} \mathrm{O}_{3}$ calcination process, Simionato and $\mathrm{Assaf}^{25}$ found that pre-existing peaks of crystalline $\mathrm{Al}_{2} \mathrm{O}_{3}$ were degraded by the loading of Co. Finally, it should be added that the annealing of pure AAO membranes at temperatures up to $500{ }^{\circ} \mathrm{C}$ can induce some changes in optical properties, e.g., photoluminescence. ${ }^{26}$

It is obvious from the shape of the hysteresis loops that the easy axis of magnetization of the samples is parallel to the wire axis and the hard axis is perpendicular to the wire axis. This agrees with the predictions of a micromagnetic model proposed by Shima et al. $^{27}$ for a cylinder of amorphous Co-P. If certain criteria concerning dimensions and magnetic exchange length are satisfied (as calculation shows them to be for our wires), then the magnetization will lie parallel to the axis of the cylinder with some spreading at the top and bottom faces. In the case of an array of Co-P cylinders, dipolar interactions are strong enough to reduce substantially the squareness - mainly because the switching field of a cylinder is comparatively low. By contrast, the fact that our CoPt arrays retain a squareness close to unity indicates that dipolar interaction between neighboring wires is not decisive: this is a consequence of the much higher switching field of a CoPt cylinder compared with a $\mathrm{Co}-\mathrm{P}$ cylinder.
The hysteresis loops of Fig. 4 are redolent of the StonerWohlfarth model, ${ }^{28}$ a not unexpected result since it is reasonable to assume that magnetization reversal will be dominated by rotational processes - the wires (assumed discrete) are too small to support domain walls. The main contribution to the total anisotropy $(K)$ is that of shape; it dominates the magnetocrystalline anisotropy in wires with this aspect ratio. In the classic Stoner-Wohlfarth model, the easy axis coercivity is $2 K / M_{\text {s }}$, i.e., the coercivity is inversely proportional to the saturation magnetization. This type of behavior is obeyed over a restricted range of annealing temperature [Figs. 3(a) and 3(b)], namely, $400{ }^{\circ} \mathrm{C}<T_{\mathrm{A}}<600{ }^{\circ} \mathrm{C}$. However, it would be simplistic to assume that the Stoner-Wohlfarth model (based on coherent rotation) should provide a complete basis for interpretation. Thus, the wires certainly contain imperfections which reduce local exchange coupling and, based on these considerations, Chen et al. ${ }^{29}$ have proposed a model of reversal whereby $H_{\mathrm{c}}$ falls with decreasing $M_{\mathrm{s}}$. This result (observed here for $T_{\mathrm{A}}>600{ }^{\circ} \mathrm{C}$ ) is predicated on discrete elliptical grains, a situation that might be provoked at this annealing temperature. On the other hand, in the temperature range $0{ }^{\circ} \mathrm{C}<T_{\mathrm{A}}<400{ }^{\circ} \mathrm{C}$, the $M_{\mathrm{s}}$ value remains constant but there is a small but monotonic increase in $H_{\mathrm{c}}$. This is not easy to explain because stress relief, likely to occur at these low temperatures (and indicated by the x-ray results), should diminish coercivity. On the other hand, lowtemperature annealing might simultaneously remove local discontinuities in exchange coupling and so promote coherent rotation. (Of course, a reversal process involving coherent rotation modes has a higher coercivity than that for the equivalent incoherent modes.) In reality, many mechanisms can influence coercivity and the observed result is not easy to predict. For example, if the hypothesised absorption of Co atoms into the $\mathrm{Al}_{2} \mathrm{O}_{3}$ pores is taken as fact, this reduces the effective magnetic diameter of a wire. This in turn changes the aspect ratio of an individual wire and enhances the easy axis coercivity.

In principle, the hard axis loop of a Stoner-Wohlfarth system is anhysteretic, a result that is independent of $M_{\mathrm{s}}$ and the magnitude of the shape anisotropy. In this respect the hard axis coercivity should be less susceptible to any microstructural changes induced by annealing. Figure 3(b) does confirm that the hard axis coercivity of our wires, although nonzero, is indeed largely independent of $T_{\mathrm{A}}$.

In conclusion, it has been demonstrated that the magnetic behavior of Co-rich cobalt platinum nanowire arrays can be improved by an appropriate annealing regime. The optimum properties required for a recording medium (e.g., coercivity and squareness) are attained at an annealing temperature of $600{ }^{\circ} \mathrm{C}$. Fortunately, this is below the temperature at which irreversible, deleterious changes occur in the microstructure of the films. A study of the composition dependence of cobalt platinum nanowire arrays is currently underway and will be reported elsewhere.

\section{ACKNOWLEDGMENTS}

The authors gratefully acknowledge Professor M. E. Pemble for providing space to prepare samples, Dr. C. A. 
Faunce for assistance with TEM measuerment, and Dr. M. Georgieva with the VSM measurement. We are grateful to Mr. Y. Liu and Miss J. Zhang for many discussions. W. Li gives special thanks to the Research Scholarship Committee of Salford for a studentship. Also, the support of EPSRC and an award from Salford University Academic Development Fund are greatly appreciated.

${ }^{1}$ R. A. Ristau, K. Barmak, L. H. Lewis, K. R. Coffey, and J. K. Howard, J. Appl. Phys. 86, 4527 (1999).

${ }^{2}$ G. A. Held, H. Zeng, and S. H. Sun, J. Appl. Phys. 95, 1481 (2004).

${ }^{3}$ T. S. Vedantam, J. P. Liu, H. Zeng, and S. Sun, J. Appl. Phys. 93, 7184 (2003).

${ }^{4}$ S. Jeong, Y. N. Hsu, D. E. Laughlin, and M. E. McHenry, IEEE Trans. Magn. 36, 2336 (2000).

${ }^{5}$ C. Tsang, M. M. Chen, and T. Yogi, Proc. IEEE 81, 1346 (1993).

${ }^{6}$ D. Y. Oh and J. K. Park, J. Appl. Phys. 93, 7756 (2003).

${ }^{7}$ M. Yu, Y. Liu, and D. J. Sellmyer, J. Appl. Phys. 87, 6959 (2000).

${ }^{8}$ J. A. Christodoulides, Y. Huang, Y. Zhang, G. C. Hadjipanayis, I. Panagiotopoulos, and D. Niarchos, J. Appl. Phys. 87, 6938 (2000).

${ }^{9}$ Y. W. Wang, L. D. Zhang, G. W. Meng, X. S. Peng, Y. X. Jin, and J. Zhang, J. Phys. Chem. B 106, 2502 (2002).

${ }^{10}$ Y. Peng, T. H. Shen, and B. Ashworth, J. Appl. Phys. 93, 7050 (2003).

${ }^{11}$ R. Ferré, K. Ounadjela, J. M. George, L. Piraux, and S. Dubois, Phys. Rev. B 56, 14066 (1997).

${ }^{12}$ Z. T. Zhang, D. A. Blom, Z. Gai, J. R. Thompson, J. Shen, and S. Dai, J. Am. Chem. Soc. 125, 7528 (2003).

${ }^{13}$ Y. H. Huang, H. Okumura, G. C. Hadjipanayis, and D. Weller, J. Appl. Phys. 91, 6829 (2002)
${ }^{14}$ N. Yasui, A. Imada, and T. Den, Appl. Phys. Lett. 83, 3347 (2003).

${ }^{15}$ Y. Peng, D. H. Qin, R. J. Zhou, and H. L. Li, Mater. Sci. Eng., B 77, 246 (2000).

${ }^{16}$ Y. Peng, H. L. Zhang, S. L. Pan, and H. L. Li, J. Appl. Phys. 87, 7405 (2000).

${ }^{17}$ D. A. Thompson and J. S. Best, IBM J. Res. Dev. 44, 311 (2000).

${ }^{18}$ H. Forster, T. Schrefl, R. Dittrich, D. Suess, W. Scholz, V. Tsiantos, J. Fidler, K. Nielsch, H. Hofmeister, H. Kronmüller, and S. Fischer, IEEE Trans. Magn. 38, 2580 (2002).

${ }^{19}$ Y. Henry, K. Ounadjela, L. Piraux, S. Dubois, J. M. George, and J. L. Duvail, Eur. Phys. J. B 20, 35 (2001).

${ }^{20}$ M. Darques, A. Encinas, L. Vila, and L. Piraux, J. Phys. D 37, 1411 (2004).

${ }^{21}$ M. Darques, A. Encinas, L. Vila, and L. Piraux, J. Phys.: Condens. Matter 16, S2279 (2004).

${ }^{22}$ J. L. Wang, X. S. Chen, G. H. Wang, B. L. Wang, W. Lu, and J. J. Zhao, Phys. Rev. B 66, 085408 (2002).

${ }^{23}$ K. B. Lee, S. M. Lee, and J. W. Cheon, Adv. Mater. (Weinheim, Ger.) 13, 517 (2001).

${ }^{24}$ Y. K. Su, D. H. Qin, H. L. Zhang, H. Li, and H. L. Li, Chem. Phys. Lett. 388, 406 (2004).

${ }^{25}$ M. Simionato and E. M. Assaf, Mater. Res. 6, 535 (2003).

${ }^{26}$ Y. Du, W. L. Cai, C. M. Mo, J. Chen, L. D. Zhang, and X. G. Zhu, Appl. Phys. Lett. 74, 2951 (1999)

${ }^{27}$ M. Shima, M. Hwang, and C. A. Ross, J. Acoust. Soc. Am. 93, 3440 (2003).

${ }^{28}$ E. C. Stoner and E. P. Wohlforth, Proc. R. Soc. London, Ser. A 240, 559 (1948).

${ }^{29}$ W. Chen, S. L. Tang, M. Lu, and Y. W. Du, J. Phys.: Condens. Matter 15, 4623 (2003). 